\title{
Adaptive Photonic Meta-surfaces Exploiting Interfacial Phase Change in Elemental Gallium
}

\author{
R. F. Waters ${ }^{1}$, K. F. MacDonald ${ }^{1 *}$, P. A. Hobson ${ }^{2}$, and N. I. Zheludev ${ }^{1,3}$ \\ ${ }^{I}$ Optoelectronics Research Centre \& Centre for Photonic Metamaterials, University of Southampton, SO17 1BJ, UK \\ ${ }^{2}$ QinetiQ Ltd., Cody Technology Park, Farnborough, GU14 OLX, UK \\ ${ }^{3}$ Centre for Disruptive Photonic Technologies, Nanyang Technological University, Singapore 637371, Singapore \\ kfm@orc.soton.ac.uk
}

\begin{abstract}
Surface-driven metallization in a nanoscale layer of elemental gallium forming the backplane of a photonic metamaterial absorber provides a mechanism for reversible all-optical and thermo-optical tuning of resonant response.

OCIS codes: (160.3918) Metamaterials; (240.6700) Surfaces
\end{abstract}

We report on the development and first experimental demonstration of a gallium-based photonic metamaterial, in which a reversible transition between solid and liquid phases occurring in a confined nanoscale surface layer of the metal drives significant changes in resonant response, offering high contrast visible/near-infrared reflectivity switching in the visible/near-infrared range that may be controlled by low intensity optical excitation.

The generic form of a resonant metamaterial absorber comprises a planar array of sub-wavelength plasmonic metal resonators and a continuous metallic (mirror) backplane, separated by a thin dielectric spacer - the resonant frequency being set by the design of the nanostructured metal layer and thickness of the spacer. Mechanisms to achieve dynamic tuning/switching of metamaterial response, i.e. frequency and/or amplitude modulation, typically rely on functional media (e.g. silicon, chalcogenide phase-change glasses, liquid crystals) placed in contact/proximity to the metal framework. Here instead we harness an interfacial structural phase transition in the metallic framework itself (the mirror plane of a resonant absorber) to drive reversible changes in spectral response (Fig. 1).

(a)
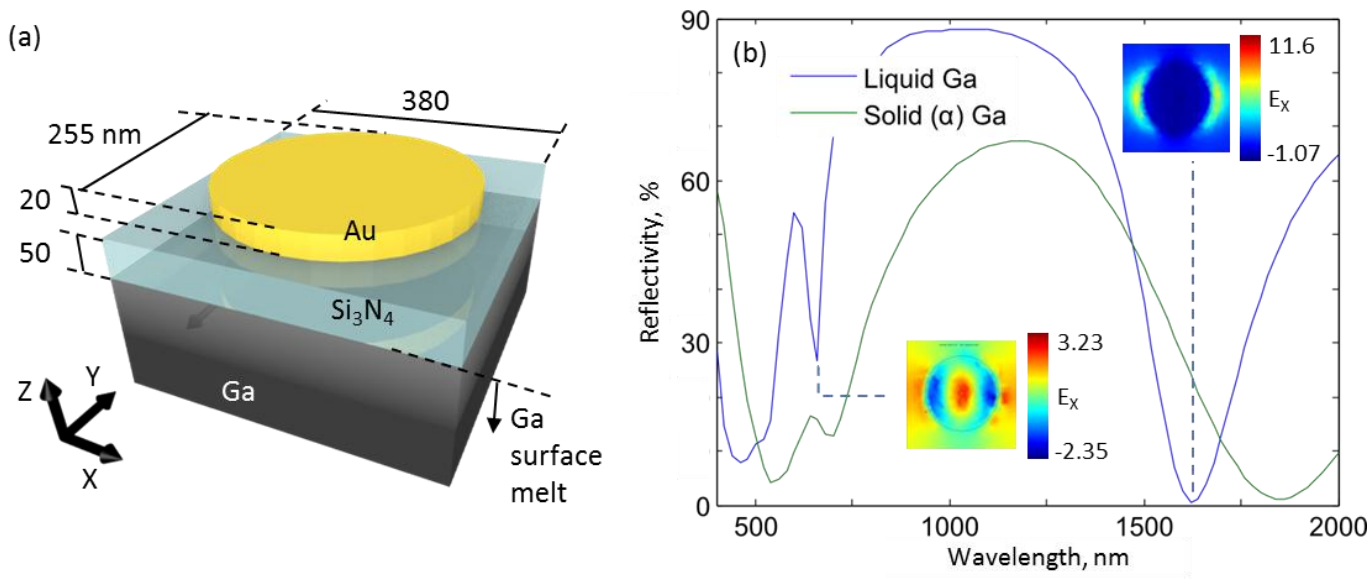

Fig. 1: (a) Artistic impression and dimensional details for numerical modeling of a gallium-backplane metamaterial absorber unit cell. (b) Numerically modeled reflectivity spectra for the structure presented in panel (a), illustrating the contrast achievable via the interfacial transition between solid and liquid gallium phases at the silicon nitride interface. Insets show the electric field distribution in the gold nano-disc plane [mid-height in the z-direction] at the absorption resonance positions indicated.

Gallium is known as a remarkably polymorphic element, existing in up to nine different phases with properties ranging from those of the 'semi-metallic' $\alpha$-phase (the stable bulk solid form) through to those of the liquid, which is highly essentially a free-electron metal. A significant change in optical properties is thus associated with its solidliquid transition, which occurs in the bulk metal at $T_{m}=29.8^{\circ} \mathrm{C}$. But gallium shows strong 'surface melting' behavior whereby a layer (only a few nanometers thick) of the highly metallic liquid phase forms at an interface between the solid $\alpha$-phase and a dielectric even at temperatures several degrees below $T_{m}$. The thickness of this 
surface layer, and thereby the optical properties of the metal/dielectric interface are highly sensitive to both temperature and incident light intensity. It has previously been shown that this surface-metallization phenomenon can underpin substantial optical nonlinearities in planar gallium mirror and gallium nanoparticle geometries [1,2]. In the present study we demonstrate that it can provide a mechanism for tuning/switching the spectral signature of (self-)adaptive photonic metamaterial systems.

We consider a meta-surface absorber formed of a gold nano-disc array, a gallium backplane and a silicon nitride spacer as illustrated in Fig. 1a. This structure is numerically modeled in fully three-dimensional finite-element Maxwell solver simulations, assuming monochromatic, plane wave illumination of the structure at normal incidence and periodic boundary conditions in the absorber plane. Figure $1 \mathrm{~b}$ shows numerically simulated reflection spectra for the solid $(\alpha)$ and liquid phases of gallium. The structural transition in gallium, which needs only to occur to a depth of several tens of nanometers for full switching of the nitride interface optical properties, brings about a substantial change in the spectral dispersion of reflectivity, perhaps most notably a >200 nm shift in the strong nearinfrared absorption resonance, providing high-contrast reflectivity switching bands across the VIS-NIR range.

Experimental samples are fabricated by depositing gold on a silicon nitride membrane, structuring the metal by focused ion beam milling to form the nano-disc pattern, and then pressing the opposing side of the membrane against a liquid gallium droplet on a thermostatic plate to form the nitride/gallium mirror plane (Fig. 2a). Reflectivity spectra are obtained by microspectrophotometry at temperatures above and several degrees below $T_{m}$ (Fig. 2b).
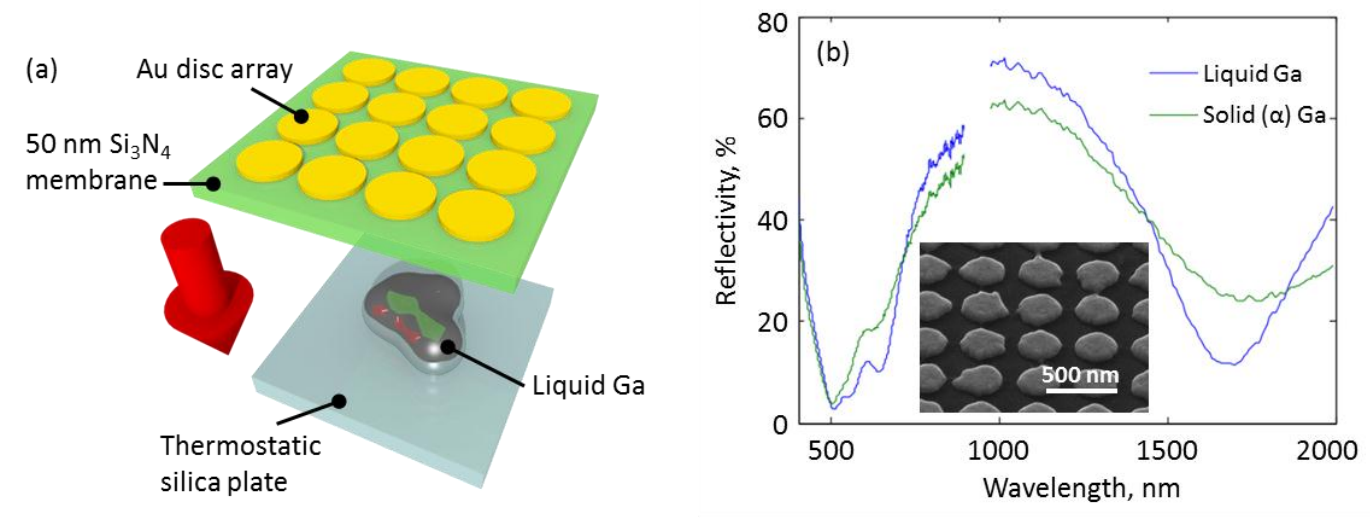

Fig. 2: (a) Schematic of the gallium meta-surface absorber fabrication procedure, whereby the phase-change backplane is formed by pressing a silicon nitride membrane, supporting a nanostructured gold film on the opposite face, into contact with liquid gallium on a thermostatic plate. (b) Experimental reflectivity spectra for the solid and liquid gallium backplane states of the meta-surface absorber shown in the inset scanning electron microscope image [oblique incidence view of the structured 30 $\mathrm{nm}$ gold layer on silicon nitride; nano-disc diameter $295 \mathrm{~mm}]$.

Ultrathin photonic meta-surfaces with actively controllable and/or self-adaptive spectral response functions offer applications potential in fields ranging from radiation emitters and sensors to spatial light modulators. As a functional material platform gallium presents the possibility of continuously tunable and switchable spectral response controlled by low-intensity $\left(\mu \mathrm{W} / \mu \mathrm{m}^{2}\right)$ light on the nanosecond timescale.

\section{References}

[1] A. V. Rode, M. Samoc, B. Luther-Davies, E. G. Gamaly, K. F. MacDonald, and N. I. Zheludev, "Dynamics of light-induced reflectivity switching in gallium films deposited on silica by pulsed laser abaltion," Opt. Lett. 26, 441-443 and 852 (2001).

[2] B. F. Soares, K. F. MacDonald, V. A. Fedotov, and N. I. Zheludev, "Light-induced switching between structural forms with different optical properties in a single gallium nanoparticulate," Nano Lett. 5, 2104-2107 (2005).

This work was supported by Dstl, through the MAST Programme, the Engineering and Physical Sciences Research Council, The Royal Society and the Ministry of Education, Singapore. 